\title{
NOTES
}

\section{Nonisothermal Crystallization Kinetics of Poly(butylene succinate) and Poly(ethylene succinate)}

\author{
Zhaobin QIU, ${ }^{* * *, \dagger}$ So FuJINAMI, ${ }^{* *}$ Motonori Komura, ${ }^{* *}$ Ken NAKAJIMA,** \\ Takayuki IKEHARA, ${ }^{*}$ and Toshio NiSHI** \\ *Department of Applied Chemistry, Faculty of Engineering, Kanagawa University, \\ 3-27-1, Rokkakubashi, Kanagawa-ku, Yokohama 221-8686, Japan \\ **Department of Organic and Polymeric Materials, Graduate School of Science and Engineering, \\ Tokyo Institute of Technology, 2-12-1 Ohokayama, Meguro-ku, Tokyo 152-8552, Japan
}

(Received February 2, 2004; Accepted May 11, 2004; Published August 15, 2004)

\begin{abstract}
KEY WORDS Poly(butylene succinate) / Poly(ethylene succinate) / Nonisothermal Crystallization Kinetics / Avrami Equation / Ozawa Equation / DSC /

[DOI 10.1295/polymj.36.642]
\end{abstract}

Much more attention has been directed to biodegradable polymers due to their potential applications in the fields related to environmental protection in the last two decades. According to the difference in preparation methods, biodegradable polymers can be classified into two types. One is the biosynthetic polymer, such as bacterial polyhydroxyalkanoates (PHAs). Among them poly(hydroxybutyrate) (PHB) is probably the most extensively studied biodegradable thermoplastic polymer. Ha et al. recently reviewed the miscibility, properties and biodegradability of blends containing either PHB or poly(3-hydroxybutyrate-cohydroxyvalerate). ${ }^{1}$ The other is the chemosynthetic polymer, such as the aliphatic polyesters. Poly(butylene succinate) (PBSU) and poly(ethylene succinate) (PES) are just two of them. The chemical structures of PBSU and PES are $\left(-\mathrm{OCH}_{2} \mathrm{CH}_{2} \mathrm{CH}_{2} \mathrm{CH}_{2} \mathrm{O}_{2} \mathrm{CCH}_{2}-\right.$ $\left.\mathrm{CH}_{2} \mathrm{CO}-\right)_{n}$ and $\left(-\mathrm{OCH}_{2} \mathrm{CH}_{2} \mathrm{O}_{2} \mathrm{CCH}_{2} \mathrm{CH}_{2} \mathrm{CO}-\right)_{n}$, respectively.

The crystal structure, crystallization and melting behaviour of PBSU have been reported in literature. ${ }^{2-4}$ Polymer blending is often performed in order to improve the physical properties and extend the application fields of PBSU. PBSU was found to be miscible with poly(vinylidene fluoride), poly(vinylidene chloride-co-vinyl chloride), poly(ethylene oxide) (PEO) and poly(vinyl phenol). ${ }^{5-9}$ On the other hand, PBSU was found to show no miscibility with PHB, poly(3hydroxybutyrate-co-hydroxyvalerate) and poly( $\varepsilon$-caprolactone). ${ }^{10-12} \mathrm{We}$ also studied the subsequent melting behavior of PBSU crystallized nonisothermally from the melt. ${ }^{13}$

The crystal structure, crystallization behaviour and melting behaviour of PES have been reported in liter- ature. ${ }^{14-16}$ The crystallization and morphology of PES in binary miscible blends of two crystalline polymers have also been reported recently, such as in PES/PHB and PES/PEO blends. ${ }^{17,18} \mathrm{We}$ also studied the subsequent melting behavior of PES crystallized nonisothermally from the melt and the crystallization kinetics as well as subsequent melting behaviour of PES from the amorphous glassy state. ${ }^{13,19}$

Crystallinity is known to play an important role in the physical properties and biodegradability of biodegradable polymers. Meanwhile, the crystalline structure and morphology of semicrystalline polymers are also influenced greatly by the thermal history. Therefore, much more attention should be paid to the crystallization kinetics study since it affects not only the crystalline structure and morphology of semicrystalline polymers but also the final physical properties and biodegradability for the biodegradable polymers. However, to the best of our knowledge, the overall crystallization kinetics studies, especially the nonisothermal crystallization kinetics studies from the melt, of PBSU and PES have not been reported so far in literature. But it is essential to study the nonisothermal crystallization kinetics from the viewpoint of practical application because most polymer processing operations are carried out under nonisothermal conditions.

In this note we reported our results on the nonisothermal crystallization kinetics of PBSU and PES from the melt by differential scanning calorimetry (DSC). It is expected that the results will be helpful for a better understanding of the relationship between structure and properties of PBSU and PES from the viewpoint of practical process.

${ }^{\dagger}$ To whom correspondence should be addressed (Tel: +81-45-481-5661×3848; E-mail: zbqiu99@yahoo.com). 


\section{EXPERIMENTAL}

$\operatorname{PBSU} \quad\left(T_{\mathrm{g}} \approx-34^{\circ} \mathrm{C}, \quad T_{\mathrm{m}} \approx 118^{\circ} \mathrm{C}\right.$ and $M_{\mathrm{w}}=$ 140000) was supplied by Showa High Polymer Co., Ltd., and PES $\left(T_{\mathrm{g}} \approx-12^{\circ} \mathrm{C}, T_{\mathrm{m}} \approx 103^{\circ} \mathrm{C}\right)$ used in this study was purchased from Aldrich Chemical Co., Inc. Nonisothermal crystallization of PBSU and PES was studied with a TA Instruments differential scanning calorimeter (DSC) 2910 equipped with a Thermal Analyst 2000. All scans were run under a nitrogen gas purge to minimize thermo-oxidative degradation. The PBSU samples were first melted at $150^{\circ} \mathrm{C}$ for $3 \mathrm{~min}$ to destroy any thermal history and then cooled at various constant cooling rates between 1 and $10{ }^{\circ} \mathrm{C} / \mathrm{min}$. In the case of PES, the annealed temperature was chosen as $130^{\circ} \mathrm{C}$, and the cooling rates varied between 1 and $5{ }^{\circ} \mathrm{C} / \mathrm{min}$.

\section{RESULTS AND DISCUSSION}

Both PBSU and PES are biodegradable semicrystalline polyesters. Their chemical structures are very similar. They are only different in their number of methylene groups between the two ether groups of the repeat unit, namely 4 and 2 for PBSU and PES, respectively.

As described in the experimental part, the PBSU samples were first melted at $150{ }^{\circ} \mathrm{C}$ for $3 \mathrm{~min}$ to destroy any previous thermal history and then cooled from the melt at various constant cooling rates to study the nonisothermal melt crystallization kinetics. In the case of PES, the annealed temperature was selected as $130^{\circ} \mathrm{C}$ since the $T_{\mathrm{m}}$ of PES was lower than that of PBSU. Figure 1 shows the DSC traces of PBSU and PES cooled from the melt at various cooling rates, from which it can be seen that the heat flow of the crystallization exotherm of PBSU and PES shifts to the low temperature range with increasing the cooling rates. It should be pointed out that PBSU could crystallize even at a cooling rate of $100^{\circ} \mathrm{C} / \mathrm{min}$, while in the case of PES the crystallization exotherm was hardly observed if the cooling rate was faster than $20^{\circ} \mathrm{C} / \mathrm{min}$. The above results indicate that the crystallizability of PBSU is higher than that of PES. The nonisothermal crystallization kinetics of PBSU and PES was studied at the cooling rate $\leq 10^{\circ} \mathrm{C} / \mathrm{min}$ in this work for the sake of the accuracy of the DSC data.

Integration of the exothermic peak during the nonisothermal scans shown in Figure 1a gave the development of relative crystallinity of PBSU as a function of temperature (Figure 2a). The plot of relative crystallinity $\left(X_{t}\right) v s$. crystallization temperature shifts to the low temperature range with the cooling rate. During the nonisothermal crystallization from the melt at

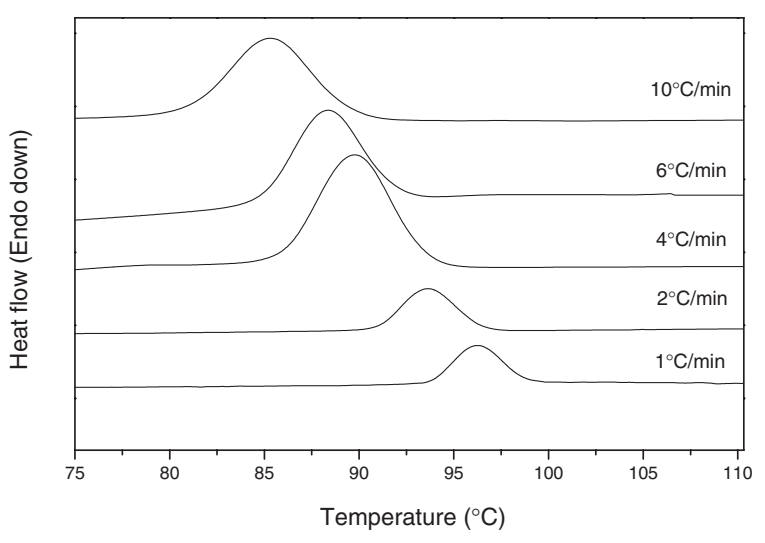

(a)

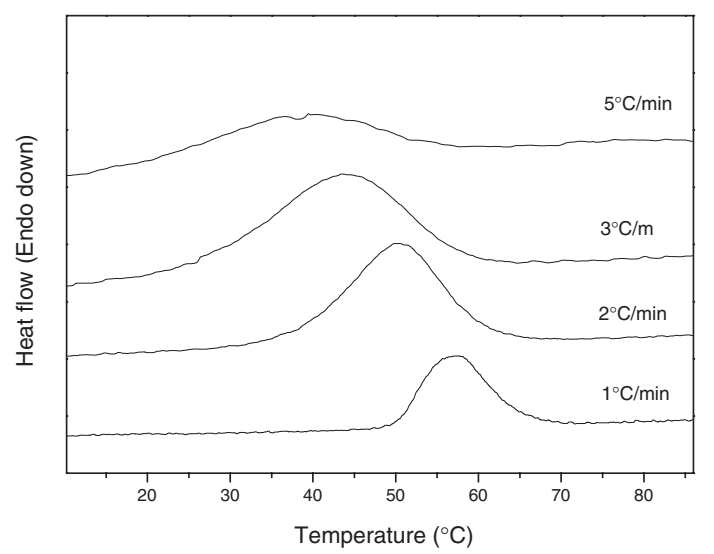

(b)

Figure 1. DSC traces cooled from the melt at various cooling rates: (a) PBSU; (b) PES. The cooling rates are indicated in the figure.

a constant cooling rate, the relationship between the crystallization time $t$ and the crystallization temperature $T$ can be described as

$$
t=\frac{T_{\mathrm{o}}-T}{\Phi}
$$

where $\Phi$ is the cooling rate, and $T_{\mathrm{o}}$ is the onset temperature of crystallization. Figure $2 \mathrm{~b}$ shows the development of relative crystallinity as a function of crystallization time by using eq 1 for PES cooled from the melt at various cooling rates. It can be seen from Figure $2 b$ that the faster the cooling rate, the shorter the crystallization time.

The well-known Avrami equation is often used to analyze the isothermal crystallization kinetics; it assumes the development of relative degree of crystallinity with crystallization time $t$ as

$$
X_{\mathrm{t}}=1-\exp \left(-k_{\mathrm{a}} t^{n_{\mathrm{a}}}\right)
$$

where $n_{\mathrm{a}}$ is the Avrami exponent depending on the nature of nucleation and growth geometry of the crys- 


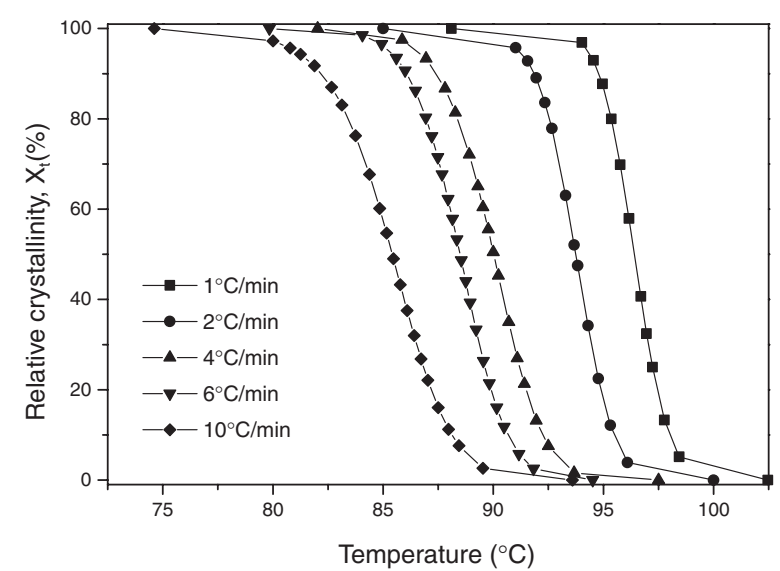

(a)

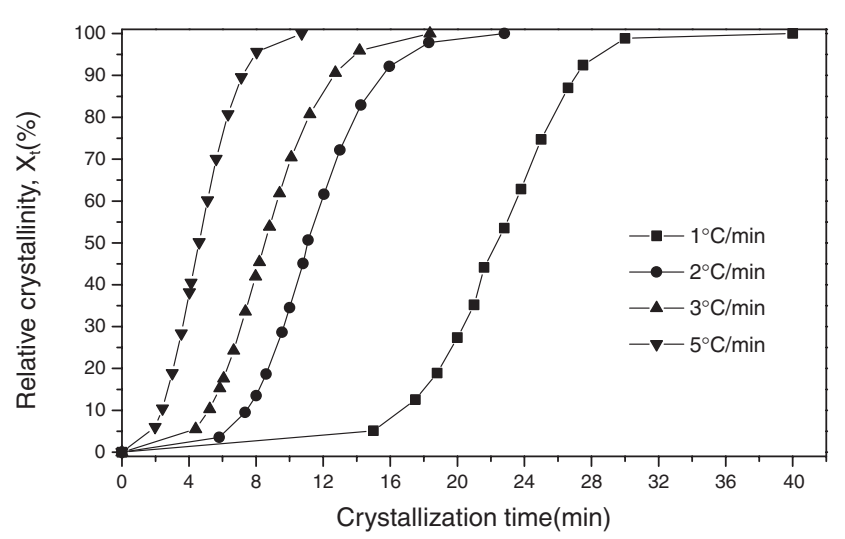

(b)

Figure 2. (a) Development of relative crystallinity as a function of crystallization temperature for PBSU during the nonisothermal crystallization from the melt and (b) development of relative crystallinity as a function of crystallization time for PES during the nonisothermal crystallization from the melt.

tals, and $k_{\mathrm{a}}$ is a composite rate constant involving both nucleation and growth rate parameters. ${ }^{20}$

Although the Avrami equation is often used to study the isothermal crystallization behavior of polymers, it has also been modified in order to describe the nonisothermal crystallization of polymers. ${ }^{21,22}$ Considering the nonisothermal character of the process investigated, Jeziorny suggested that the value of the rate parameter $k_{\mathrm{a}}$ should be adequately corrected. ${ }^{21}$ The factor that should be considered was the cooling rate, $\Phi$. Assuming constant or approximately constant $\Phi$, the final form of the parameter $k_{\mathrm{c}}$ characterizing the kinetics of nonisothermal crystallization was given as $\log k_{\mathrm{c}}=\left(\log k_{\mathrm{a}}\right) / \Phi$.

Using eq 2 in its double-logarithmic form, we could plot $\log \left(-\ln \left(1-X_{\mathrm{t}}\right)\right)$ against $\log t$ for each cooling rate as shown in Figure $3 a$ and $b$ for PBSU and PES, respectively. A series of almost straight lines

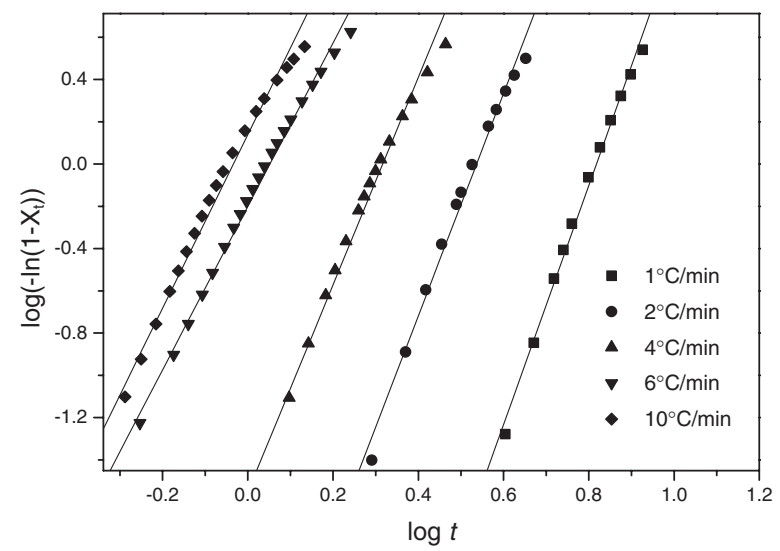

(a)

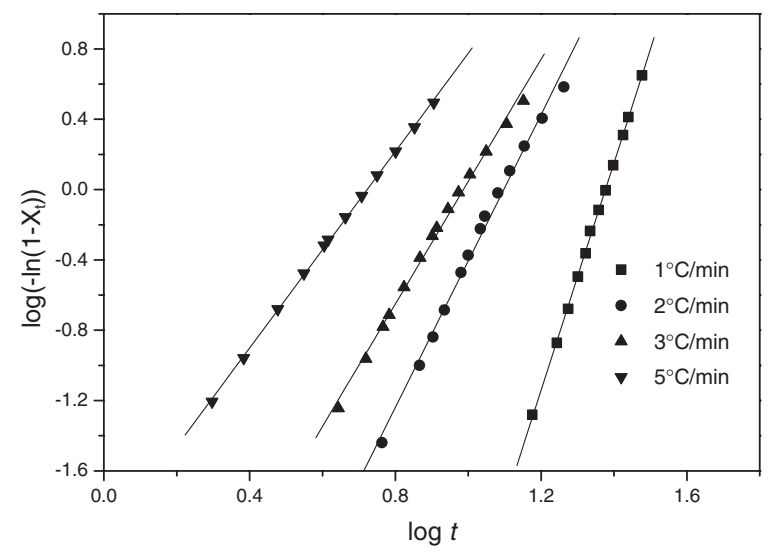

(b)

Figure 3. Avrami plots of PBSU and PES during the nonisothermal crystallization from the melt: (a) PBSU; (b) PES.

Table I. Nonisothermal crystallization kinetics parameters of PBSU from the melt based on the Avrami equation modified by Jeziorny

\begin{tabular}{ccc}
\hline $\begin{array}{c}\Phi \\
\left({ }^{\circ} \mathrm{C} / \mathrm{min}\right)\end{array}$ & $n_{\mathrm{a}}$ & $\begin{array}{c}k_{\mathrm{c}} \\
\left(\mathrm{min}^{-n_{\mathrm{a}}}\right)\end{array}$ \\
\hline 1 & 5.66 & $2.34 \times 10^{-5}$ \\
2 & 5.26 & $3.89 \times 10^{-2}$ \\
4 & 4.91 & $4.10 \times 10^{-1}$ \\
6 & 3.87 & $9.27 \times 10^{-1}$ \\
10 & 4.10 & 1.03 \\
\hline
\end{tabular}

were obtained, from which the values of $n_{\mathrm{a}}$ and $k_{\mathrm{a}}$ were obtained. The values of $k_{\mathrm{c}}$ were obtained by modifying $k_{\mathrm{a}}$ with $\Phi$. The values of $n_{\mathrm{a}}$ and $k_{\mathrm{c}}$ are listed in Tables I and II for PBSU and PES, respectively. The value of $n_{\mathrm{a}}$ decreases with increasing the cooling rate for both PBSU and PES. The decrease of $n_{\mathrm{a}}$ is more significant in PES than that in PBSU. On the other hand, the value of $k_{\mathrm{c}}$ increases with increasing the cooling rate for both PBSU and PES. At the same cooling rate, the crystallization rate of PES is slower 
Table II. Nonisothermal crystallization kinetics parameters of PES from the melt based on the Avrami equation modified by Jeziorny

\begin{tabular}{ccc}
\hline $\begin{array}{c}\Phi \\
\left({ }^{\circ} \mathrm{C} / \mathrm{min}\right)\end{array}$ & $n_{\mathrm{a}}$ & $\begin{array}{c}k_{\mathrm{c}} \\
\left(\mathrm{min}^{-n_{\mathrm{a}}}\right)\end{array}$ \\
\hline 1 & 6.46 & $1.29 \times 10^{-9}$ \\
2 & 4.16 & $5.19 \times 10^{-3}$ \\
3 & 3.48 & $7.13 \times 10^{-2}$ \\
5 & 2.88 & $3.94 \times 10^{-1}$ \\
\hline
\end{tabular}

than that of PBSU, indicating again that the crystallizability of PBSU is higher than that of PES. It can be concluded that the Avrami equation modified by Jeziorny was able to describe the nonisothermal crystallization process of both PBSU and PES.

Ozawa extended the Avrami equation for isothermal crystallization to the nonisothermal case by assuming that the sample was cooled with a constant rate from the molten state. ${ }^{22}$ In the Ozawa method, the time variable in the Avrami equation is replaced by a cooling rate and the relative crystallinity is derived as a function of constant cooling rate $\Phi$ as:

$$
X_{\mathrm{t}}=1-\exp \left(\frac{-K(T)}{\Phi^{n_{\mathrm{o}}}}\right)
$$

where $K(T)$ is the cooling (or heating) function at crystallization temperature $T$ and $n_{\mathrm{o}}$ is the Ozawa exponent which depends on the type of nucleation and growth mechanism. Double logarithms of eq 3 and rearrangement results in the following form:

$$
\log \left(-\ln \left(1-X_{\mathrm{t}}\right)\right)=\log K(T)-n_{\mathrm{o}} \log \Phi
$$

If the Ozawa equation describes the nonisothermal crystallization process very well, a series of straight lines of $\log \left(-\ln \left(1-X_{\mathrm{t}}\right)\right)$ against $\log \Phi$ should be obtained for the chosen crystallization temperature. The Ozawa equation is found to be able to describe the nonisothermal crystallization process of poly(ethylene terephthalate) well. ${ }^{22}$ The Ozawa plots of PBSU and PES are shown in Figure 4. Figure 4a shows the Ozawa plots of PBSU cooled from the melt at various cooling rates, from which it was found that the Ozawa method failed to describe the nonisothermal crystallization process of PBSU as shown by the curves rather than straight lines. On the other hand, Figure $4 b$ shows the Ozawa plots of $\log \left(-\ln \left(1-X_{\mathrm{t}}\right)\right) v s . \log \Phi$ for PES, from which it is found that the Ozawa equation could describe the nonisothermal crystallization process well, and the exponent $n_{\mathrm{o}}$ as well as the cooling function $K(T)$ were obtained and listed in Table III. The cooling function $K(T)$ is found to decrease with increasing the crystallization temperature. The Ozawa exponent $n_{\mathrm{o}}$ varied between 1.6 and 2.6 in the range of crystallization temperature of 35 to $55^{\circ} \mathrm{C}$, with the average value being around 2 . In the previous

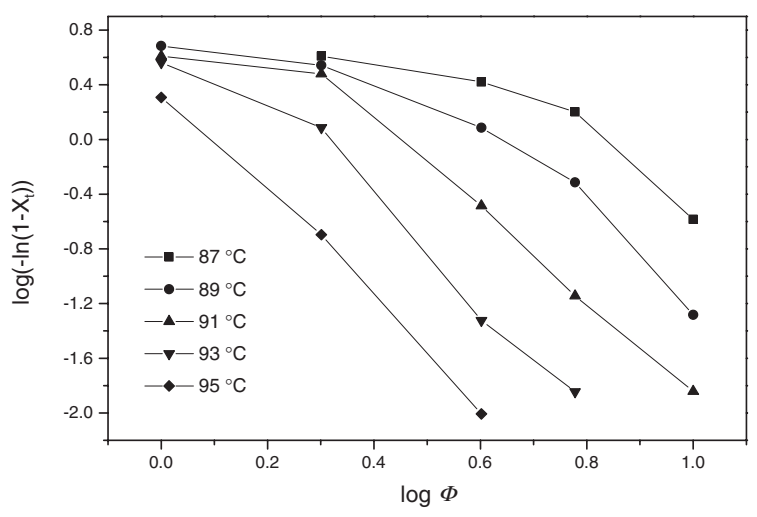

(a)

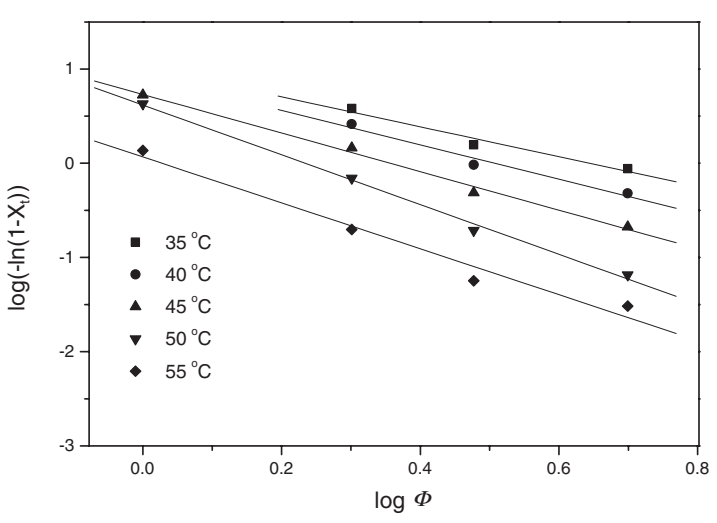

(b)

Figure 4. Ozawa plots of PBSU and PES during the nonisothermal crystallization from the melt: (a) PBSU; (b) PES.

Table III. Nonisothermal crystallization kinetics parameters of PES from the melt based on the Ozawa equation

\begin{tabular}{ccc}
\hline $\begin{array}{c}\text { Crystallization temperature } \\
\left({ }^{\circ} \mathrm{C}\right)\end{array}$ & $n_{\mathrm{o}}$ & $\begin{array}{c}K(T) \\
\left({ }^{\circ} \mathrm{C} / \mathrm{min}\right)^{n_{\mathrm{o}}}\end{array}$ \\
\hline 35 & 1.59 & $3.89 \times 10^{1}$ \\
40 & 1.83 & 8.43 \\
45 & 2.05 & 5.37 \\
50 & 2.64 & 4.15 \\
55 & 2.44 & 1.17 \\
\hline
\end{tabular}

work, we also studied the nonisothermal crystallization kinetics of PES from the amorphous glassy state by DSC and analyzed with the Ozawa equation. ${ }^{19}$ The Ozawa method was also able to describe the nonisothermal crystallization process from the amorphous glassy state; however, the Ozawa exponent $n_{\mathrm{o}}$ varied between 3 and 4 for the crystallization temperature range of 22.5 to $32.5^{\circ} \mathrm{C}$. The difference in the values of $n_{\mathrm{o}}$ suggests that the crystallization mechanism should be different, which resulted from the difference in the initial stage of the crystallization process, namely one from the melt state and the other from the 
glassy state.

Now let us discuss the possible reason that the Ozawa equation was able to describe the nonisothermal crystallization process of PES but failed to describe that of PBSU. This may be related to the following fact that the secondary crystallization of PBSU was more apparent than that of PES. As shown in Figure 3a, the Avrami plots of PBSU were found to deviate from the straight lines at the late stage, indicative of the occurrence of the secondary crystallization. But the similar results have not been observed in the case of PES (Figure 3b). The Ozawa method was also found to fail to describe the nonisothermal crystallization process of poly(aryl ether ketones) (PAEKs). The reason was ascribed to the secondary crystallization of PAEKs. ${ }^{23}$ Generally, the crystallization of polymers consists of two processes, namely, primary crystallization and secondary crystallization. If the secondary crystallization is apparent, the Avrami equation can not describe the whole crystallization process. Two sets of Avrami parameters are required to fit the primary crystallization and secondary crystallization, respectively, in such cases. However, Ozawa took no account of the secondary crystallization and assumed that the cooling function and Ozawa exponent were constant over the entire crystallization process. ${ }^{22}$ Therefore, the Ozawa method often fails to describe the nonisothermal crystallization process in the case of the occurrence of the secondary crystallization. This reason holds true for PBSU in this work.

\section{CONCLUSIONS}

Nonisothermal melt crystallization kinetics of two biodegradable polyesters PBSU and PES was studied with DSC for the first time in this work. It was found that the Avrami equation modified by Jeziorny could describe the nonisothermal crystallization process of both PBSU and PES. With increasing the cooling rate, the crystallization rate of both PBSU and PES increased. Meanwhile, the Avarami exponent, $n_{\mathrm{a}}$, decreased with the increase of the cooling rate for both PBSU and PES. For PBSU the value of $n_{\mathrm{a}}$ decreased from 5.66 to 4.1 when the cooling rate increased from 1 to $10^{\circ} \mathrm{C} / \mathrm{min}$. For PES the value of $n_{\mathrm{a}}$ decreased from 6.46 to 2.88 when the cooling rate increased from 1 to $5{ }^{\circ} \mathrm{C} / \mathrm{min}$. The above results indicated that the cooling rate had a more apparent influence on the nonisothermal crystallization behavior of PES than that of PBSU. The Ozawa equation was also employed to study the nonisothermal crystallization process of PBSU and PES. The Ozawa method failed to describe the nonisothermal crystallization kinetics of PBSU from the melt, while it could describe that of PES well. The failure of the application of the Ozawa method to the nonisothermal crystallization process of PBSU may be related to the secondary crystallization.

Acknowledgment. Z. Qiu thanks the Japan Society for the Promotion of Science for providing the fellowship and Grant-in-Aid (P01278) to do this research at Tokyo Institute of Technology, Tokyo, Japan.

\section{REFERENCES}

1. C. S. Ha and W. J. Cho, Prog. Polym. Sci., 27, 759 (2002).

2. Y. Chatani, R. Hasegawa, and H. Tadokoro, Polym. Prepr., Jpn., 20, 420 (1971).

3. T. Miyata and T. Masuko, Polymer, 39, 1399 (1998).

4. E. S. Yoo and S. S. Im, J. Polym. Sci., Polym. Phys. Ed., 37, 1357 (1999).

5. J. C. Lee, H. Tazawa, T. Ikehara, and T. Nishi, Polym. J., 30, 327 (1998).

6. J. C. Lee, H. Tazawa, T. Ikehara, and T. Nishi, Polym. J., 30, 780 (1998).

7. Z. B. Qiu, T. Ikehara, and T. Nishi, Polymer, 44, 2799 (2003).

8. Z. B. Qiu, T. Ikehara, and T. Nishi, Polymer, 44, 3095 (2003).

9. Z. B. Qiu, M. Komura, T. Ikehara, and T. Nishi, Polymer, 44, 8111 (2003).

10. Z. B. Qiu, T. Ikehara, and T. Nishi, Polymer, 44, 2503 (2003).

11. Z. B. Qiu, T. Ikehara, and T. Nishi, Polymer, 44, 7519 (2003).

12. Z. B. Qiu, M. Komura, T. Ikehara, and T. Nishi, Polymer, 44, 7749 (2003).

13. Z. B. Qiu, M. Komura, T. Ikehara, and T. Nishi, Polymer, 44, 7781 (2003).

14. A. S. Ueda, Y. Chatani, and H. Tadokoro, Polym. J., 2, 387 (1971).

15. Y. Ichikawa, J. Washiyama, Y. Moteki, K. Noguchi, and K. Okuyama, Polym. J., 27, 1264 (1995).

16. I. A. Al-Raheil and A. M. A. Qudah, Polym. Int., 37, 249 (1995).

17. H. A. Al-Salah, Polym. Bull., 41, 593 (1998).

18. Z. B. Qiu, T. Ikehara, and T. Nishi, Macromolecules, 35, 8251 (2002).

19. Z. B. Qiu, T. Ikehara, and T. Nishi, Polymer, 44, 5429 (2003).

20. M. Avrami, J. Chem. Phys., 7, 1103 (1939).

21. A. Jeziorny, Polymer, 19, 1142 (1978).

22. T. Ozawa, Polymer, 12, 150 (1971).

23. Z. B. Qiu, H. W. Zhou, Z. S. Mo, H. F. Zhang, and Z. W. Wu, Polym. J., 32, 287 (2000). 